\title{
From Static Structures to Continuous Conformational Changes on the Energy Landscapes
}

\author{
Ghoncheh Mashayekhi, Ali Dashti, Peter Schwander and Abbas Ourmazd \\ University of Wisconsin-Milwaukee, Milwaukee, Wisconsin, United States
}

The dynamical behavior of a wide variety of systems depends on the energy landscape explored by the system. We apply machine learning approaches to experimental data in order to determine the dynamical behavior of biological nanomachines over their energy landscapes. Using particles in equilibrium and without any time resolution, this approach promises the possibility to find functional, minimum-energy paths, and compile movies of the associated continuous conformational changes on the energy landscape.

The key lies in recognizing that many structures co-exist in an ensemble, each with a sizable probability. The underlying reason is the fact that the hierarchy of energy barriers between the structures are easily surmountable by thermal fluctuations under physiological conditions [1-4]. Under such circumstances, a structure is a point in the continuum of conformations, each with a different free energy. This means that the energy landscape of biomolecular conformations consists ofhills and valleys $[\underline{3}, 4, \underline{5}]$. In an ensemble of molecules in equilibrium, the probability of finding a specific conformation drops exponentially with the free energy of that conformation. Therefore, such energy landscapes can be compiled by counting the frequency with which each conformation occurs in an ensemble of molecules in equilibrium [1, $\underline{6,7]}$ To compile the energy landscape, one must have the snapshots of individual members of the ensemble. This rules out techniques, which average over conformations and constitutes a decisive advantage for so-called single-particle methods like cryogenic electron microscopy (cryo-EM).

The examination of energy landscape derived from the experimental snapshots offers substantial information beyond static structures. Importantly, it can reveal the path of least-action representing the most probable sequence of transitions between any two states in the landscape.Compiling the conformational changes the biological machine undergoes along such a trajectory offers important insights into biological function.

\section{Acknowledgement}

The research is in collaboration with the Data Science group (UWM), Frank et al (Columbia), des George et al (CUNY), and Singharoy et al (ASU).

The research conducted at UWM was supported by the US Department of Energy, Office of Science, Basic Energy Sciences, under contract DE-SC0002164 (A.O., algorithm design and development) and by the US National Science Foundation (NSF) under contract STC 1231306 (A.O., numerical trial models and data analysis; M.S., data analysis) and under contract number 1551489 (A.O., underlying analytical models).

\section{References}

1. Dashti, A. et al. Proc. Natl. Acad. Sci. USA 111, 17492-17497 (2014).

2. Henzler-Wildman, K. \& Kern, D. Nature 450, 964-972 (2007).

3. Ramachandran, G. N., Ramakrishnan, C. \& Sasisekharan, V. J. Mol. Biol. 7, 95-99 (1963).

4. Frauenfelder, H., Sligar, S. G. \& Wolynes, P. G. Science 254, 1598-1603 (1991). 
5. Fischer, N., Konevega, A. L., Wintermeyer, W., Rodnina, M. V. \& Stark, H. Nature 466, 329-333 (2010).

6. Frank, J. \& Ourmazd, A. Methods 100, 61-67 (2016).

7. Dashti, A. et al. Preprint at https://doi.org/10.1101/291922 (2019) 